\title{
Assessment of Shoot and Fruit Damage on Number Basis in Okra
}

\author{
A.R. Lakhamapure ${ }^{1}$, Sh.N.V. Lavhe ${ }^{1 *}$, P.S. Barde ${ }^{1}$, R.O. Deotale ${ }^{1}$ and \\ P.R. Panchabhai ${ }^{2}$ \\ ${ }^{1}$ Entomology Section, College of Agriculture, Nagpur, India \\ ${ }^{2}$ Entomology Section, College of Agriculture, Akola, India \\ *Corresponding author
}

\section{A B S T R A C T}

\section{Keywords}

Abelmoschus esculentus,

Earias vittella, Shoot

infestation, Number basis

Article Info

Accepted:

24 August 2018

Available Online:

10 December 2018
To assess the shoot/fruit infestation and damage (number basis) on okra was undertaken during kharif season of 2016-17 in field of Insectory premises of Entomology Section, College of Agriculture, Nagpur. The trial incorporating nine treatments including control which were replicated thrice in Randomized Block Design. During this investigation, on the basis of above parameters revealed that, lowest infestation (shoot and fruit infestation) and highest yield over control was observed in treatment of Spinosad $45 \mathrm{SC} 0.01 \%\left(\mathrm{~T}_{8}\right.$; $6.52 \%$ ) shoot infestation, $12.83 \%$ fruit infestation and $16.13 \%$ fruit damage with 86.79 $\mathrm{q} /$ ha yield followed by Emamectin benzoate $5 \mathrm{SG} 0.02 \%\left(\mathrm{~T}_{7}\right)$, Indoxacarb $15.5 \mathrm{EC} 0.01 \%$ $\left(\mathrm{T}_{5}\right)$, Acetamiprid $20 \mathrm{SP} 0.2 \mathrm{~g} / \mathrm{lit}\left(\mathrm{T}_{6}\right)$ Bacillus thuringensis $2 \mathrm{ml} / 1\left(\mathrm{~T}_{4}\right)$, Neem oil $2 \%\left(\mathrm{~T}_{1}\right)$, NSE 5\% $\left(\mathrm{T}_{3}\right)$ and Karanj oil $2 \%\left(\mathrm{~T}_{2}\right)$.

\section{Introduction}

Okra (Abelmoschus esculentus L.) is one of the most popular vegetable crop grown extensively all over India. However, summer and kharif are the favourable seasons for its cultivation. The area under okra cultivation in India is $4,09,000$ ha with production of $41,93,000$ metric tonnes. Whereas, in Maharashtra State it is cultivated on $26,2,030.3$ ha area with production of 1 , 65,900.4 metric tonnes (Anonymous, 2015).

Among the various insect pests, sucking pests like leafhopper, Amrasca biguttula biguttula Ishida and whitefly, Bemisia tabaci Gennadius pose a major threat affecting the okra production.The damage due to fruit borer accounts for nearly $45 \%$ in Karnataka, $22.5 \%$ in Uttar Pradesh, 25.93-40.91\% in Madhya Pradesh and in Rajasthan it was estimated that if insecticidal protection was not given there would be a net yield loss of $54.04 \%$ and repeated use of insecticides has resulted in the development of resistance in the insect pest and disturbance to the agro- ecosystem by affecting the non-target pests and somewhere its indirect effect found on fruit/ shoot infestation and damage Hence, in the present study we recorded the number of infested shoots in each plot a day before first spray and 3,7 and 14 days after each spraying on five randomly selected plants from each plot and recorded number of infested fruits and number of marketable fruits on five randomly selected plants from each net plot picking wise. In 
order to know the per cent fruit infestation. The fruits were plucked from 5 selected plants, they were counted Similarly, from those total fruits the infested fruits due to fruit borer were separated and counted.

\section{Materials and Methods}

The present field experiment was carried out on Okra crop, variety Akola bahar during kharif season 2016-2017 in the Insectory field of Entomology Section, College of Agriculture, Nagpur.

The experiment was laid out in a randomized block design with Nine treatments consisting Neem oil 2\% ( $\left.\mathrm{T}_{1}\right)$, Karanj oil 2\% ( $\left.\mathrm{T}_{2}\right)$, NSE $5 \%\left(\mathrm{~T}_{3}\right)$, Bacillus thuringensis $2 \mathrm{ml} / 1 \quad\left(\mathrm{~T}_{4}\right)$, Indoxacarb $15.5 \mathrm{EC} 0.01 \%\left(\mathrm{~T}_{5}\right)$, Acetamiprid 20 SP $0.2 \mathrm{~g} /$ lit $\left(\mathrm{T}_{6}\right)$, Emamectin benzoate $5 \mathrm{SG}$ $0.02 \% \quad\left(\mathrm{~T}_{7}\right), \quad$ Spinosad $45 \quad \mathrm{SC} 0.01 \%(\mathrm{~T} 8)$ including (T9) control (water spray) were evaluated after each spray against Natural enemies and observed the effect on them.

The plot size was kept $12.6 \mathrm{~m}^{2}$ with a spacing of $60 \times 45 \mathrm{~cm}$ between rows and plants respectively and recommended agronomical practices were followed.

And the efficacy of each treatment was determined on the basis of per cent shoot infestation, fruit infestation (on number basis) by shoot and fruit borer. The cumulative (average) per cent of shoot and fruit infestation and fruit damage was calculated to see the overall effect of various treatments. With the help of following formula

Number of infested shoots Per cent shoot infestation $=$--------------- $\times 100$

Total number of shoots

Per cent fruit
Infestation (Number basis) $\times 100$

\section{Results and Discussion}

Total number of fruits

Cumulative per cent shoot infestation by shoot and fruit borer

Shoot infestation was noticed at 30 DAS and continue upto 65 DAS. The data on the cumulative per cent shoot infestation presented in Table 1 and illustrated in figure 1. From the results, it was revealed that, all the treatments were significantly superior over control in reducing the shoot infestation.

Among these different treatments, Spinosad $45 \mathrm{SC} 0.01 \%\left(\mathrm{~T}_{8}\right)$ recorded minimum $(6.52 \%)$ shoot infestation and was found to be the best followed by Emamectin benzoate 5 SG $0.02 \%$ $\left(\mathrm{T}_{7} ; 7.09 \%\right)$ and Indoxacarb 15.8 EC $0.01 \%$ $\left(\mathrm{T}_{5} ; 7.50 \%\right)$. Devi et al., (2014) reported $5.00 \%$ shoot infestation with the treatment spinosad $45 \%$ SC and emamectin benzoate $5 \%$ SG $5.50 \%$.

The next effective treatment in reducing per cent shoot infestation was of Acetamiprid 20 SP $0.2 \mathrm{~g} / \mathrm{lit}\left(\mathrm{T}_{6} ; 7.92 \%\right)$ and it was followed by Bacillus (B.t.) $\left(1 \times 10^{8} \mathrm{CFU}\right) 2 \mathrm{ml} / \mathrm{lit}\left(\mathrm{T}_{4}\right.$; $8.51 \%$ ). Puranik et al., (2002) reported $11.07 \%$ shoot infestation of $L$. orbonalis with the treatment halt WP $(B t)$.

The next promising treatment in reducing per cent shoot infestation was of Neem oil $2 \%\left(\mathrm{~T}_{1}\right.$; $8.83 \%)$ followed by NSE $5 \%\left(\mathrm{~T}_{3} ; 8.94 \%\right)$.

Both these treatments were statistically at par with each other. Our present findings regarding efficacy of neem oil $2 \% \quad\left(\mathrm{~T}_{5}\right)$ confirmed the findings of Panzade (2006), who recorded $7.95 \%$, shoot infestation with the treatment of neem oil $1 \%$.

The Karanj oil 2\% ( $\left.\mathrm{T}_{2} ; 9.06 \%\right)$ showed least effectiveness in reducing per cent shoot 
infestation but found to be superior over control $\left(\mathrm{T}_{9} ; 10.29 \%\right)$.

Per cent fruit infestation by shoot and fruit borer, 3, 7 and 14 days after first spray (On number basis)

The data pertaining to per cent fruit infestation indicated (Table 2) that, all the treatments were significantly superior over control in suppressing fruit borer infestation. 3 DAT, the treatment of Spinosad 45 SC $0.01 \%\left(\mathrm{~T}_{8}\right)$ recorded the lowest (18.40\%) infestation followed by Emamectin benzoate 5 SG $0.02 \%$ $\left(\mathrm{T}_{7} ; 19.65 \%\right)$, Indoxacarb $15.8 \mathrm{EC} 0.01 \%\left(\mathrm{~T}_{5}\right.$; $20.20 \%$ ), Acetamiprid 20 SP 0.2 g/lit $\left(\mathrm{T}_{6}\right.$; $20.35 \%$ ) and these treatments were statistically at par with the treatment of Spinosad 45 SC $0.01 \%\left(\mathrm{~T}_{1}\right)$. Priya and Mishra (2007) reported $7.74 \%$ fruit borer infestation with the treatment of spinosad 45\% SC. Anil and Sharma (2010) recorded lowest fruit infestation with emamectin benzoate 5 SG $0.002 \%$.

The next effective treatment was of Bacillus thuringensis $2 \mathrm{ml} / \mathrm{l}\left(\mathrm{T}_{4}\right)$, which recorded $21.80 \%$ fruit infestation followed by Neem oil $2 \%\left(\mathrm{~T}_{1} ; 22.10 \%\right)$ and NSE $5 \%\left(\mathrm{~T}_{3} ; 23.01\right)$, Karanj oil $2 \%\left(\mathrm{~T}_{2} ; 23.90 \%\right)$ and all these treatments were statistically at par with each other.

7 days after first spray, the treatment Spinosad $45 \mathrm{SC} \quad 0.01 \% \quad\left(\mathrm{~T}_{8}\right)$ recorded the lowest $(19.65 \%)$ infestation followed by Emamectin benzoate 5 SG $0.02 \% \quad\left(\mathrm{~T}_{7} ; 20.55 \%\right)$, Indoxacarb 15.8 EC $0.01 \%\left(\mathrm{~T}_{5} ; 21.05 \%\right)$, Acetamiprid 20 SP $0.2 \mathrm{~g} / \mathrm{lit}\left(\mathrm{T}_{6} ; 21.08 \%\right)$ and these treatment were statistically at par with the treatment of Spinosad $45 \mathrm{SC} 0.01 \%\left(\mathrm{~T}_{8}\right)$.

The next promising treatments were of Bacillus thuringensis $2 \mathrm{ml} / \mathrm{l}\left(\mathrm{T}_{4} ; 22.04 \%\right)$, Neem oil $2 \%\left(\mathrm{~T}_{1} ; 22.80 \%\right)$, NSE $5 \%\left(\mathrm{~T}_{3}\right.$; $23.60 \%)$ and Karanj oil $2 \%\left(\mathrm{~T}_{2} ; 25.60 \%\right)$. The treatment regarding Karanj oil 2\% $\quad\left(\mathrm{T}_{2}\right)$ showed higher infestation $27.06 \%$ but found to be superior over control $\left(\mathrm{T}_{9}\right)$, where $31.60 \%$ infestation was recorded. Puranik et al., (2002) reported $11.78 \%$ fruit infestation with Dipel 8L.

Similar type of results were obtained 14 days after first spray in which the treatment Spinosad of 45 SC $0.01 \%\left(\mathrm{~T}_{8}\right)$ recorded the lowest $(21.15 \%)$ infestation followed by Emamectin benzoate 5 SG $0.02 \% \quad\left(\mathrm{~T}_{7}\right.$; $21.45 \%)$, Indoxacarb $15.8 \mathrm{EC} \quad 0.01 \% \quad\left(\mathrm{~T}_{5}\right.$; $21.85 \%)$, Acetamiprid $20 \mathrm{SP} 0.2 \mathrm{~g} / \mathrm{lit}\left(\mathrm{T}_{6}\right.$; $21.90 \%$ ) and these treatments were statistically at par with the treatment of Spinosad $45 \mathrm{SC} 0.01 \%\left(\mathrm{~T}_{8}\right)$.

In botanicals and biopesticide promising treatments were of Bacillus thuringensis $2 \mathrm{ml} / 1$ $\left(\mathrm{T}_{4} ; 22.84 \%\right)$, Neem oil $2 \%\left(\mathrm{~T}_{1} ; 22.90 \%\right)$, NSE $5 \%\left(\mathrm{~T}_{3} ; 23.80 \%\right)$ and these treatments were statistically at par with the treatment of Bacillus thuringensis $2 \mathrm{ml} / 1\left(\mathrm{~T}_{4}\right)$. The treatment regarding Karanj oil 2\% $\left(\mathrm{T}_{2}\right)$ showed higher infestation $28.01 \%$ but found to be superior over control $\left(\mathrm{T}_{9}\right)$, where $31.60 \%$ infestation was recorded.

As the results on this parameter are also studied after second and third spray the results are not discussed here. They are discussed further in results obtained after second and third spray.

\section{Per cent fruit infestation by shoot and fruit borer, 3, 7 and 14 days after second spraying (on number basis)}

The results reported in Table 2 revealed that, all the treatments were found to be superior over control in reducing per cent fruit infestation, 3 DAT. The treatment of Spinosad $45 \mathrm{SC} 0.01 \%\left(\mathrm{~T}_{8}\right)$ recorded the lowest fruit infestation (8.40\%).

The next effective treatment in reducing per cent fruit infestation was of Emamectin 
benzoate $5 \quad \mathrm{SG} \quad 0.02 \% \quad\left(\mathrm{~T}_{7} ; 21.10 \%\right)$, Indoxacarb 15.8 EC $0.01 \% \quad\left(\mathrm{~T}_{5} ; 21.19 \%\right)$ followed by Acetamiprid 20 SP $0.2 \mathrm{~g} / \mathrm{lit}$, Bacillus thuringensis $2 \mathrm{ml} / \mathrm{l} \quad\left(\mathrm{T}_{4} ; 23.80 \%\right)$, Neem oil $2 \%\left(\mathrm{~T}_{1} ; 24.80 \%\right)$ NSE $5 \%\left(\mathrm{~T}_{3}\right.$; $25.80 \%)$ and Karanj oil 2\% ( $\left.\mathrm{T}_{2} ; 27.10 \%\right)$. Priya and Mishra (2007) reported $7.74 \%$ fruit borer infestation with the treatment of spinosad 45\% SC. Anil and Sharma (2010) recorded lowest fruit infestation with emamectin benzoate $5 \mathrm{SG} 0.02 \%$ and Bajad $e t$ al., (2011) registered lowest fruit damage $27.13 \%$ with Indoxacarb $0.007 \%$. In treatment of Karanj oil $\left(\mathrm{T}_{2}\right)$ somewhat higher per cent fruit infestation of $27.10 \%$ was recorded but the treatment was found to be superior over control $\left(\mathrm{T}_{8} ; 32.30 \%\right)$. Similar results on per cent fruit infestation were obtained 7 days after second spray and are discussed below.
The results showed that, the treatment of Spinosad 45 SC $0.01 \%\left(\mathrm{~T}_{8}\right)$ recorded lowest fruit infestation $(9.50 \%)$.

The next promising treatments were of Emamectin benzoate $5 \quad \mathrm{SG} \quad 0.02 \% \quad\left(\mathrm{~T}_{7}\right.$; $21.15 \%)$, Indoxacarb 15.8 EC $0.01 \% \quad\left(\mathrm{~T}_{5}\right.$; $23.54 \%)$, Acetamiprid 20 SP $2 \mathrm{~g} / \mathrm{lit}\left(\mathrm{T}_{6}\right.$; 23.54) Bacillus thuringensis $2 \mathrm{ml} / \mathrm{l} \quad\left(\mathrm{T}_{7}\right.$; $23.90 \%)$, and Neem oil $2 \%\left(\mathrm{~T}_{1} ; 25.12 \%\right)$ and all these treatments were statistically at par with the treatment of Emamectin benzoate 5 SG $0.02 \%\left(\mathrm{~T}_{7}\right)$. Malik and Lal (1989) reported the effect of neem oil $1 \%$ and their findings are comparable with the results obtained during this study. Singh et al., (1998) recorded $27.97 \%$ fruit infestation of E.vittella 8 DAT with the treatment of $B t$ which corroborate with the present findings.

Plate.1 Shoot infested by Earias vittella

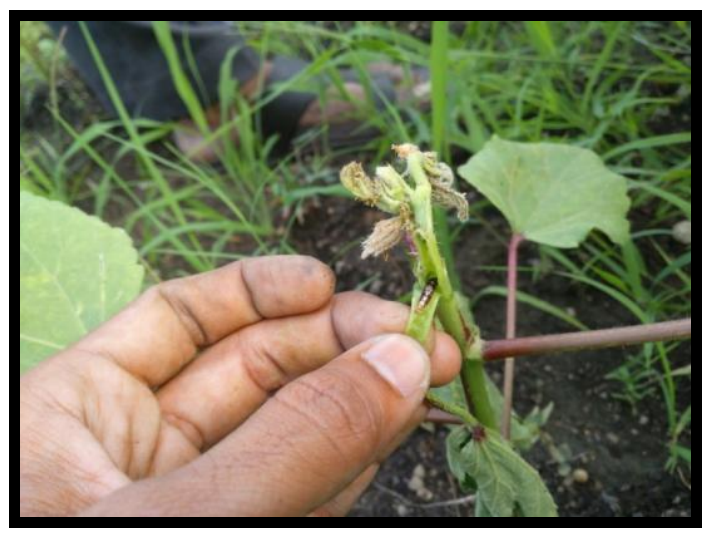

Plate.2 Fruit infested by Earias vittella

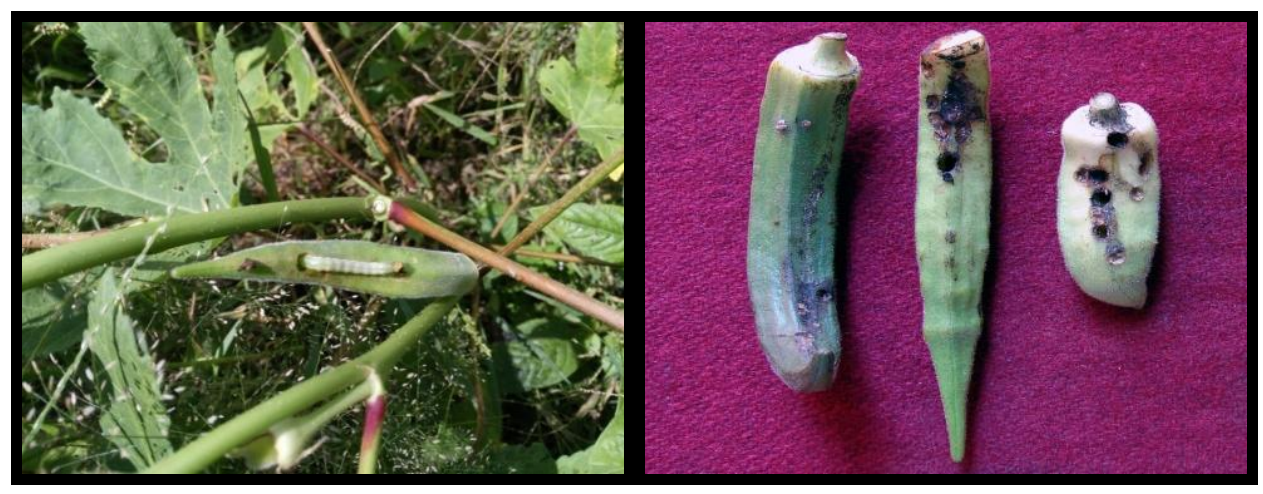


Table.1 Cumulative per cent shoot infestation by shoot and fruit borer

\begin{tabular}{|c|c|c|c|c|c|}
\hline Tr. No. & Treatments & R-I & R-II & R-III & Mean \\
\hline $\mathbf{T}_{1}$ & Neem oil & $\begin{array}{c}9.24 \\
(3.03)\end{array}$ & $\begin{array}{c}8.13 \\
(2.85)\end{array}$ & $\begin{array}{c}8.94 \\
(2.98)\end{array}$ & $\begin{array}{c}8.83 \\
(2.97)\end{array}$ \\
\hline $\mathbf{T}_{\mathbf{2}}$ & Karanj oil & $\begin{array}{c}9.82 \\
(3.13)\end{array}$ & $\begin{array}{c}8.40 \\
(2.89)\end{array}$ & $\begin{array}{c}8.97 \\
(2.99)\end{array}$ & $\begin{array}{c}9.06 \\
(3.00)\end{array}$ \\
\hline $\mathbf{T}_{\mathbf{3}}$ & Neem Seed Extract & $\begin{array}{c}9.55 \\
(3.09)\end{array}$ & $\begin{array}{c}8.33 \\
(2.88)\end{array}$ & $\begin{array}{c}8.95 \\
(2.99)\end{array}$ & $\begin{array}{c}8.94 \\
(2.98)\end{array}$ \\
\hline$\overline{T_{4}}$ & Bacillus (B.t.) $\left(1 \times 10^{8} \mathrm{CFU}^{)}\right.$ & $\begin{array}{c}9.01 \\
(3.00)\end{array}$ & $\begin{array}{c}8.35 \\
(2.88)\end{array}$ & $\begin{array}{c}8.19 \\
(2.86)\end{array}$ & $\begin{array}{c}8.51 \\
(2.91)\end{array}$ \\
\hline$\overline{T_{5}}$ & Indoxacarb 15.8 E.C & $\begin{array}{c}7.18 \\
(2.67)\end{array}$ & $\begin{array}{c}7.77 \\
(2.78)\end{array}$ & $\begin{array}{c}7.55 \\
(2.74)\end{array}$ & $\begin{array}{c}7.50 \\
(2.73)\end{array}$ \\
\hline $\mathrm{T}_{6}$ & Acetamiprid 20 S.P & $\begin{array}{c}8.13 \\
(2.85)\end{array}$ & $\begin{array}{c}7.47 \\
(2.73)\end{array}$ & $\begin{array}{c}8.18 \\
(2.86)\end{array}$ & $\begin{array}{c}7.92 \\
(2.81)\end{array}$ \\
\hline $\mathbf{T}_{7}$ & Emamectine Benzoate $5 \mathrm{SG}$ & $\begin{array}{c}6.64 \\
(2.57)\end{array}$ & $\begin{array}{c}7.56 \\
(2.74)\end{array}$ & $\begin{array}{c}7.08 \\
(2.66)\end{array}$ & $\begin{array}{c}7.09 \\
(2.66)\end{array}$ \\
\hline $\mathbf{T}_{8}$ & Spinosad 45 SC & $\begin{array}{c}6.19 \\
(2.48)\end{array}$ & $\begin{array}{c}6.77 \\
(2.60)\end{array}$ & $\begin{array}{c}6.60 \\
(2.56)\end{array}$ & $\begin{array}{c}6.52 \\
(2.55)\end{array}$ \\
\hline $\mathbf{T}_{9}$ & Control (Water spray) & $\begin{array}{l}10.30 \\
(3.20)\end{array}$ & $\begin{array}{l}10.07 \\
(3.17)\end{array}$ & $\begin{array}{l}10.50 \\
(3.24)\end{array}$ & $\begin{array}{c}10.29 \\
(3.20)\end{array}$ \\
\hline "F" test & \multicolumn{5}{|c|}{ Sig. } \\
\hline S.E.(m) \pm & \multicolumn{5}{|c|}{0.26} \\
\hline C.D at $5 \%$ & \multicolumn{5}{|c|}{0.78} \\
\hline
\end{tabular}

(Figures in parenthesis are square root transformed values.) 
Table.2 Mean per cent fruit infestation by shoot and fruit borer (on number basis)

\begin{tabular}{|c|c|c|c|c|c|c|c|c|c|}
\hline \multirow[t]{2}{*}{ Treatments } & \multicolumn{3}{|c|}{$1^{\text {st }}$ spray } & \multicolumn{3}{|c|}{$2^{\text {nd }}$ spray } & \multicolumn{3}{|c|}{$3^{\text {rd }}$ spray } \\
\hline & 3DAT & 7DAT & 14DAT & 3DAT & 7DAT & 14DAT & 3DAT & 7DAT & 14DAT \\
\hline Neem oil & $\begin{array}{l}22.10 \\
(28.04)\end{array}$ & $\begin{array}{c}22.80 \\
(28.52)\end{array}$ & $\begin{array}{l}22.90 \\
(28.59)\end{array}$ & $\begin{array}{c}24.80 \\
(29.86)\end{array}$ & $\begin{array}{c}25.12 \\
(30.07)\end{array}$ & $\begin{array}{c}25.82 \\
(30.53)\end{array}$ & $\begin{array}{c}24.50 \\
(29.66)\end{array}$ & $\begin{array}{c}25.09 \\
(30.05)\end{array}$ & $\begin{array}{c}25.75 \\
(30.49)\end{array}$ \\
\hline Karanj oil & $\begin{array}{l}23.90 \\
(29.26)\end{array}$ & $\begin{array}{c}25.60 \\
(30.39)\end{array}$ & $\begin{array}{c}28.01 \\
(31.95)\end{array}$ & $\begin{array}{l}27.10 \\
(31.37)\end{array}$ & $\begin{array}{c}28.85 \\
(32.48)\end{array}$ & $\begin{array}{l}30.10 \\
(33.27)\end{array}$ & $\begin{array}{c}26.10 \\
(30.72)\end{array}$ & $\begin{array}{c}27.80 \\
(31.82)\end{array}$ & $\begin{array}{c}30.01 \\
(33.21)\end{array}$ \\
\hline Neem Seed Extract & $\begin{array}{c}23.01 \\
(28.66)\end{array}$ & $\begin{array}{l}23.60 \\
(29.06)\end{array}$ & $\begin{array}{c}23.80 \\
(29.19)\end{array}$ & $\begin{array}{c}25.80 \\
(30.52)\end{array}$ & $\begin{array}{c}26.30 \\
(30.85)\end{array}$ & $\begin{array}{l}27.70 \\
(31.75)\end{array}$ & $\begin{array}{c}24.70 \\
(29.80)\end{array}$ & $\begin{array}{c}26.10 \\
(30.72)\end{array}$ & $\begin{array}{c}27.50 \\
(31.62)\end{array}$ \\
\hline $\begin{array}{c}\text { Bacillus (B.t.) }\left(1 \times 10^{8}\right. \\
\text { CFU) }\end{array}$ & $\begin{array}{l}21.80 \\
(27.83)\end{array}$ & $\begin{array}{c}22.04 \\
(27.99)\end{array}$ & $\begin{array}{c}22.84 \\
(28.54)\end{array}$ & $\begin{array}{l}23.80 \\
(29.19)\end{array}$ & $\begin{array}{l}24.10 \\
(29.40)\end{array}$ & $\begin{array}{l}24.50 \\
(29.66)\end{array}$ & $\begin{array}{l}22.80 \\
(28.52)\end{array}$ & $\begin{array}{l}23.90 \\
(29.26)\end{array}$ & $\begin{array}{c}24.10 \\
(29.40)\end{array}$ \\
\hline Indoxacarb 15.8 E.C & $\begin{array}{c}20.20 \\
(26.70)\end{array}$ & $\begin{array}{c}21.05 \\
(27.30)\end{array}$ & $\begin{array}{c}21.85 \\
(27.86)\end{array}$ & $\begin{array}{c}22.20 \\
(28.11)\end{array}$ & $\begin{array}{c}23.54 \\
(29.02)\end{array}$ & $\begin{array}{c}22.80 \\
(28.52)\end{array}$ & $\begin{array}{c}21.85 \\
(27.86)\end{array}$ & $\begin{array}{c}22.10 \\
(28.04)\end{array}$ & $\begin{array}{c}22.77 \\
(28.50)\end{array}$ \\
\hline Acetamiprid 20 S.P & $\begin{array}{c}20.35 \\
(26.81)\end{array}$ & $\begin{array}{l}21.08 \\
(27.33)\end{array}$ & $\begin{array}{l}21.90 \\
(27.90)\end{array}$ & $\begin{array}{l}23.20 \\
(28.79)\end{array}$ & $\begin{array}{c}23.54 \\
(29.02)\end{array}$ & $\begin{array}{l}22.80 \\
(28.52)\end{array}$ & $\begin{array}{c}23.10 \\
(28.72)\end{array}$ & $\begin{array}{c}23.35 \\
(28.89)\end{array}$ & $\begin{array}{c}22.90 \\
(28.59)\end{array}$ \\
\hline $\begin{array}{c}\text { Emamectine Benzoate } 5 \\
\text { SG }\end{array}$ & $\begin{array}{c}19.60 \\
(26.27)\end{array}$ & $\begin{array}{c}20.55 \\
(26.95)\end{array}$ & $\begin{array}{c}21.45 \\
(27.59)\end{array}$ & $\begin{array}{c}21.10 \\
(27.34)\end{array}$ & $\begin{array}{c}21.15 \\
(27.38)\end{array}$ & $\begin{array}{c}21.20 \\
(27.41)\end{array}$ & $\begin{array}{c}19.95 \\
(26.52)\end{array}$ & $\begin{array}{c}21.10 \\
(27.34)\end{array}$ & $\begin{array}{c}21.20 \\
(27.41)\end{array}$ \\
\hline Spinosad 45 SC & $\begin{array}{c}18.40 \\
(25.40)\end{array}$ & $\begin{array}{c}19.65 \\
(26.31)\end{array}$ & $\begin{array}{c}21.15 \\
(27.38)\end{array}$ & $\begin{array}{c}8.40 \\
(16.84)\end{array}$ & $\begin{array}{c}9.50 \\
(17.95)\end{array}$ & $\begin{array}{c}11.70 \\
(20.00)\end{array}$ & $\begin{array}{c}7.80 \\
(16.21)\end{array}$ & $\begin{array}{c}8.50 \\
(16.95)\end{array}$ & $\begin{array}{c}10.70 \\
(19.09) \\
\end{array}$ \\
\hline Control (Water spray) & $\begin{array}{c}30.10 \\
(33.27)\end{array}$ & $\begin{array}{c}31.60 \\
(34.20)\end{array}$ & $\begin{array}{c}32.80 \\
(34.93)\end{array}$ & $\begin{array}{c}32.81 \\
(34.94)\end{array}$ & $\begin{array}{c}33.50 \\
(35.36)\end{array}$ & $\begin{array}{c}34.15 \\
(35.75)\end{array}$ & $\begin{array}{c}36.10 \\
(36.92)\end{array}$ & $\begin{array}{c}38.70 \\
(38.46)\end{array}$ & $\begin{array}{c}40.10 \\
(39.28)\end{array}$ \\
\hline F Test & Sig & Sig & Sig & Sig & Sig & Sig & Sig & Sig & Sig \\
\hline $\operatorname{SEm}( \pm)$ & 1.41 & 1.46 & 1.51 & 1.62 & 1.57 & 1.54 & 1.51 & 1.58 & 1.63 \\
\hline CD@5\% & 4.23 & 4.38 & 4.52 & 4.86 & 4.70 & 4.62 & 4.53 & 4.74 & 4.88 \\
\hline
\end{tabular}

(Figures in parenthesis are arcs in transformed values.) 
Table.3 Cumulative per cent fruit infestation by shoot and fruit borer (On number basis)

\begin{tabular}{|c|c|c|c|c|c|}
\hline Tr. No. & Treatments & R-I & R-II & R-III & Mean \\
\hline $\mathbf{T}_{1}$ & Neem oil & $\begin{array}{c}24.34 \\
(29.56)\end{array}$ & $\begin{array}{c}22.85 \\
(28.55)\end{array}$ & $\begin{array}{c}26.64 \\
(31.07)\end{array}$ & $\begin{array}{c}24.61 \\
(29.74)\end{array}$ \\
\hline $\mathbf{T}_{2}$ & Karanj oil & $\begin{array}{c}25.48 \\
(30.31)\end{array}$ & $\begin{array}{c}30.67 \\
(33.62)\end{array}$ & $\begin{array}{c}29.75 \\
(33.05)\end{array}$ & $\begin{array}{c}28.63 \\
(32.34)\end{array}$ \\
\hline $\mathbf{T}_{3}$ & Neem Seed Extract & $\begin{array}{c}24.64 \\
(29.76)\end{array}$ & $\begin{array}{c}25.27 \\
(30.17)\end{array}$ & $\begin{array}{c}27.15 \\
(31.40)\end{array}$ & $\begin{array}{c}25.68 \\
(30.44)\end{array}$ \\
\hline $\mathbf{T}_{4}$ & Bacillus (B.t.) $\left(1 \times 10^{8} \mathrm{CFU}^{)}\right.$ & $\begin{array}{c}22.34 \\
(28.20)\end{array}$ & $\begin{array}{c}22.08 \\
(28.02)\end{array}$ & $\begin{array}{c}26.56 \\
(31.02)\end{array}$ & $\begin{array}{c}21.86 \\
(27.87)\end{array}$ \\
\hline $\mathbf{T}_{5}$ & Indoxacarb 15.8 E.C & $\begin{array}{c}21.38 \\
(27.54)\end{array}$ & $\begin{array}{c}20.98 \\
(27.26)\end{array}$ & $\begin{array}{c}21.18 \\
(27.40)\end{array}$ & $\begin{array}{c}22.97 \\
(28.63)\end{array}$ \\
\hline $\mathbf{T}_{6}$ & Acetamiprid 20 S.P & $\begin{array}{c}21.93 \\
(27.92)\end{array}$ & $\begin{array}{c}22.02 \\
(27.98)\end{array}$ & $\begin{array}{c}23.21 \\
(28.80)\end{array}$ & $\begin{array}{c}22.38 \\
(28.23)\end{array}$ \\
\hline $\mathbf{T}_{7}$ & Emamectine Benzoate $5 \mathrm{SG}$ & $\begin{array}{c}18.39 \\
(25.39)\end{array}$ & $\begin{array}{c}20.85 \\
(27.16)\end{array}$ & $\begin{array}{c}19.77 \\
(26.39)\end{array}$ & $\begin{array}{c}19.67 \\
(26.32)\end{array}$ \\
\hline $\mathbf{T}_{8}$ & Spinosad 45 SC & $\begin{array}{c}13.54 \\
(21.59)\end{array}$ & $\begin{array}{c}13.02 \\
(21.15)\end{array}$ & $\begin{array}{c}11.93 \\
(20.20)\end{array}$ & $\begin{array}{c}12.83 \\
(20.98)\end{array}$ \\
\hline $\mathbf{T}_{9}$ & Control (Water spray) & $\begin{array}{c}33.67 \\
(35.46)\end{array}$ & $\begin{array}{c}36.99 \\
(37.45)\end{array}$ & $\begin{array}{c}32.57 \\
(34.79)\end{array}$ & $\begin{array}{c}34.41 \\
(35.91)\end{array}$ \\
\hline "F" test & \multicolumn{5}{|c|}{ Sig. } \\
\hline S.E.(m) \pm & \multicolumn{5}{|c|}{0.96} \\
\hline C.D at $5 \%$ & \multicolumn{5}{|c|}{2.90} \\
\hline
\end{tabular}

(Figures in parenthesis are arcs in transformed values.) 
Fig.1 Cumulative per cent shoot infestation by shoot and fruit borer

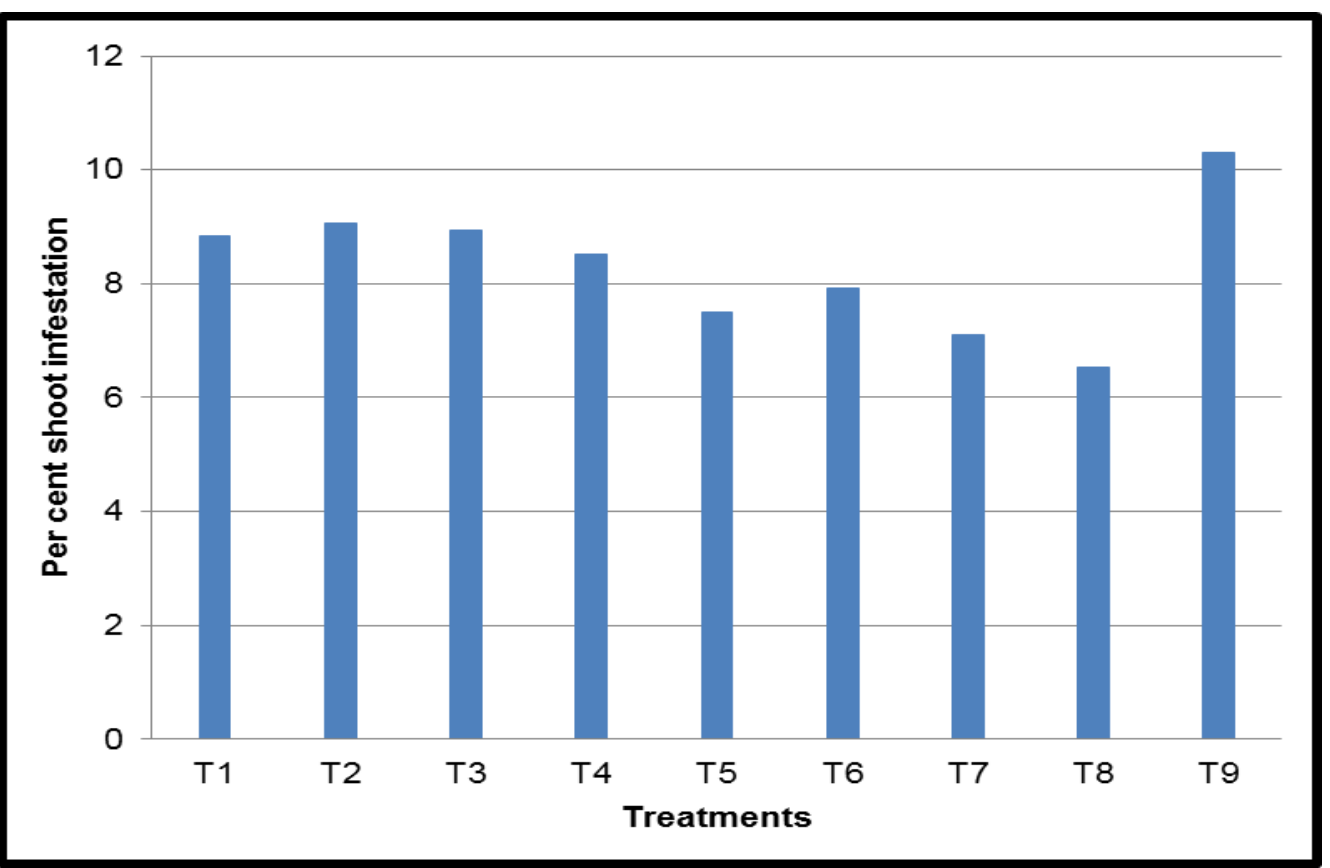

$\mathrm{T}_{1}=$ Neem oil $-2 \% ; \mathrm{T}_{2}=$ Karanj oil $-2 \% ; \mathrm{T}_{3}=\mathrm{NSE}-5 \% ; \mathrm{T}_{4}=$ Bacillus thuringiensis $-2 \mathrm{ml} / \mathrm{lit} ; \mathrm{T}_{5}=$ Indoxacarb $15.8 \mathrm{EC}-0.01 \% ; \mathrm{T}_{6}=$ Acetamiprid $20 \mathrm{SP}-0.2 \mathrm{~g} / \mathrm{lit} ; \mathrm{T}_{7}=$ Emamectin Benzoate $5 \mathrm{SG}-0.02 \% ; \mathrm{T}_{8}=$ Spinosad 45 $\mathrm{SC}-0.01 \% ; \mathrm{T}_{9}=$ Control (water spray)

Fig.2 Cumulative per cent fruit infestation by shoot and fruit borer (on number basis)

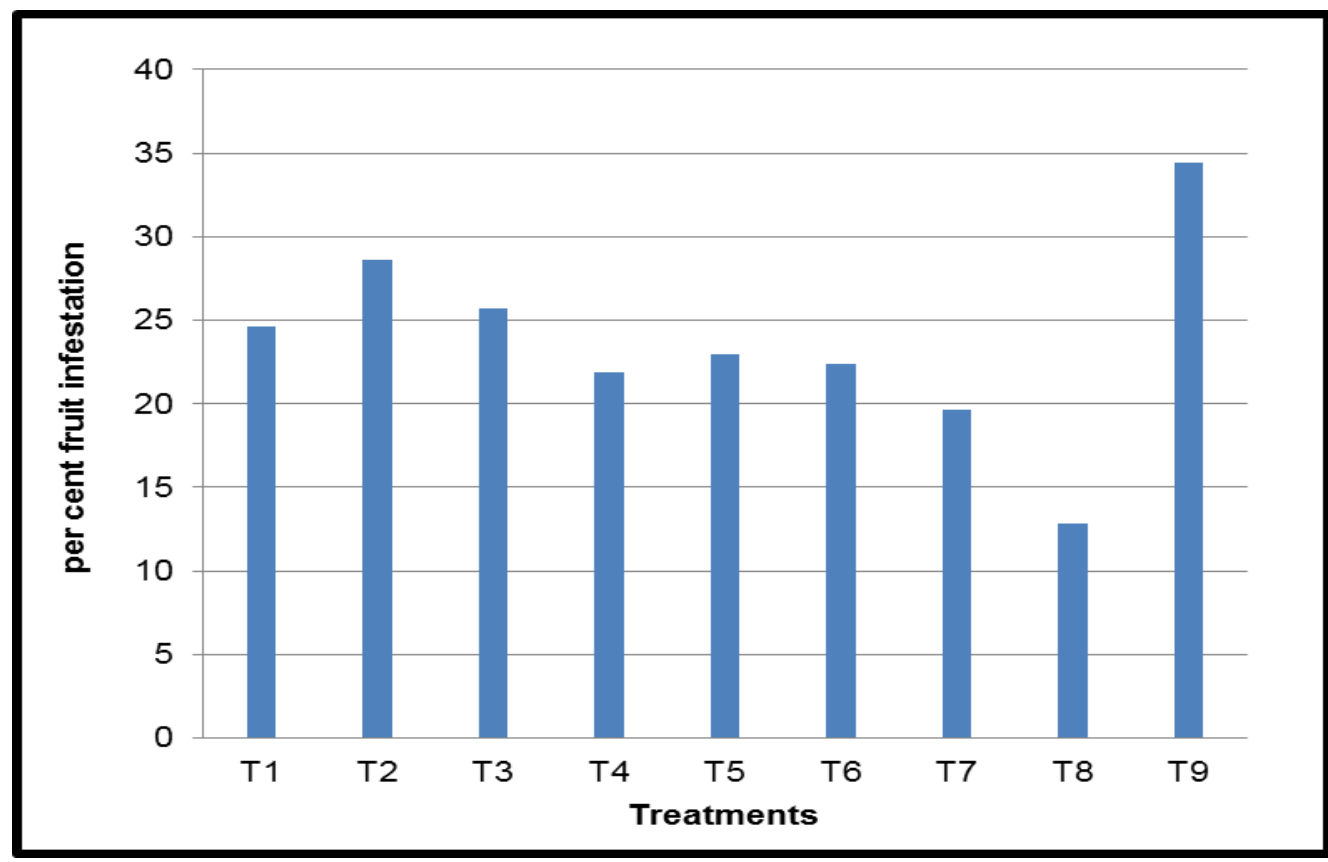

$\mathrm{T}_{1}=$ Neem oil $-2 \% ; \mathrm{T}_{2}=$ Karanj oil $-2 \% ; \mathrm{T}_{3}=\mathrm{NSE}-5 \% ; \mathrm{T}_{4}=$ Bacillus thuringiensis $-2 \mathrm{ml} /$ lit; $\mathrm{T}_{5}=$ Indoxacarb $15.8 \mathrm{EC}-0.01 \% ; \mathrm{T}_{6}=$ Acetamiprid $20 \mathrm{SP}-0.2 \mathrm{~g} / \mathrm{lit} ; \mathrm{T}_{7}=$ Emamectin Benzoate $5 \mathrm{SG}-0.02 \% ; \mathrm{T}_{8}=$ Spinosad 45 $\mathrm{SC}-0.01 \% ; \mathrm{T}_{9}=$ Control (water spray) 
The treatment of Karanj oil 2\% $\left(\mathrm{T}_{2}\right)$ recorded $28.85 \%$ fruit infestation but found to be superior over control $\left(\mathrm{T}_{9} ; 33.50 \%\right)$. Reddy and Srinivasa (2001) recorded $11.40 \%$ fruit damage with pungam oil $2 \%$.

Similar types of results were obtained 14 days after second spray in which the treatment of Spinosad 45 SC $0.01 \% \quad\left(\mathrm{~T}_{8}\right)$ recorded the lowest $(11.70 \%)$ infestation. Next promising treatment was of Emamectin benzoate $5 \mathrm{SG}$ $0.02 \%\left(\mathrm{~T}_{7} ; 21.20 \%\right)$ followed by Indoxacarb 15.8 EC $0.01 \%\left(\mathrm{~T}_{5} ; 22.80 \%\right)$, Acetamiprid 20 SP $0.2 \mathrm{~g} / \mathrm{lit}\left(\mathrm{T}_{6} ; 22.80 \%\right)$ and these treatments were statistically at par with the treatment of Emamectin benzoate $5 \mathrm{SG} 0.02 \%\left(\mathrm{~T}_{7}\right)$.

In botanicals and biopesticide promising treatments were of Bacillus thuringensis 2 $\mathrm{ml} / \mathrm{l}\left(\mathrm{T}_{4} ; 24.50 \%\right)$, Neem oil $2 \%\left(\mathrm{~T}_{1} ; 25.82 \%\right)$, NSE $5 \%\left(\mathrm{~T}_{3} ; 27.70 \%\right)$ and these treatments were statistically at par with the treatment of Bacillus thuringensis $2 \mathrm{ml} / \mathrm{l} \quad\left(\mathrm{T}_{4}\right)$. The treatment regarding Karanj oil 2\% $\left(\mathrm{T}_{2}\right)$ showed higher infestation $30.10 \%$ but found to be superior over control $\left(\mathrm{T}_{9}\right)$, where $34.15 \%$ infestation was recorded.

Per cent fruit infestation by shoot and fruit borer, 3, 7 and 14 days after third spray (on number basis)

It was evident from that, all treatments were superior over control in minimizing the shoot and fruit borer infestation, 3 DAT. The lowest infestation of fruit borer was recorded with the treatment of Spinosad $45 \mathrm{SC} 0.01 \%\left(\mathrm{~T}_{8}\right.$; $7.80 \%)$. The second best treatment was of Emamectin benzoate 5 SG $0.02 \% \quad\left(\mathrm{~T}_{7}\right.$; $19.95 \%)$ and third one was of Indoxacarb 15.8 EC $0.01 \%\left(\mathrm{~T}_{5} ; 21.85 \%\right)$.

The next effective treatments were of Acetamiprid 20 SP $0.2 \mathrm{~g} / \mathrm{lit}\left(\mathrm{T}_{6} ; 23.10 \%\right)$ Bacillus thuringensis 2ml/1 ( $\left.\mathrm{T}_{4} ; 22.80 \%\right)$, Neem oil $2 \%\left(\mathrm{~T}_{1} ; 24.50\right)$, NSE $5 \% \quad\left(\mathrm{~T}_{3}\right.$;
$24.70 \%)$ and Karanj oil $2 \%\left(\mathrm{~T}_{2} ; 26.10 \%\right)$ and all these treatments were equally effective in reducing fruit infestation. The result of NSE $5 \%\left(\mathrm{~T}_{3}\right)$ confirm the finding of Ambekar et al., (2000). They recorded $27.25 \%$ infestation of $E$. vittella. The result of neem oil $2 \%\left(\mathrm{~T}_{1}\right)$ confirm the findings of Malik and Lal (1989). The result on efficacy of Bacillus thuringensis $2 \mathrm{ml} / \mathrm{l}\left(\mathrm{T}_{4}\right)$ confirm the findings of Kharbade et al., (1999).

The treatment of Karanj oil $\left(\mathrm{T}_{2} ; 26.10 \%\right)$ was found to be least effective but found to be superior over control $\left(\mathrm{T}_{8} ; 36.10 \%\right)$. The results on Karanj oil $\left(\mathrm{T}_{2}\right)$ confirm the findings of Srinivasa rao et al., (2002) and Reddy and Srinivasa (2001).

The data pertaining to per cent fruit infestation 7 days after third spray indicated that, all the treatments were superior over control in suppressing the fruit borer infestation. The treatment Spinosad $45 \mathrm{SC}$ $0.01 \%\left(\mathrm{~T}_{8}\right)$ recorded lowest $(8.50 \%)$ fruit infestation followed by Emamectin benzoate 5 SG $0.02 \% \quad\left(\mathrm{~T}_{7} ; 21.10 \%\right)$. Next best treatments were of Indoxacarb $15.8 \mathrm{EC} 0.01 \%$ $\left(\mathrm{T}_{5} ; 22.10 \%\right)$ and Acetamiprid $20 \mathrm{SP} 0.2 \mathrm{~g} / \mathrm{lit}$ $\left(\mathrm{T}_{6} ; 23.35 \%\right)$ and these treatments were found to be statistically at par with each other.

The next effective treatments were of Bacillus thuringensis $2 \mathrm{ml} / \mathrm{l}\left(\mathrm{T}_{4} ; 23.90 \%\right)$, Neem oil $2 \%$ $\left(\mathrm{T}_{1} ; 25.09 \%\right)$, NSE $5 \%\left(\mathrm{~T}_{3} ; 26.10 \%\right)$ and Karanj oil $2 \%\left(\mathrm{~T}_{2} ; 27.80 \%\right)$ and all these treatments were statistically comparable with each other. The findings of NSE 5\% $\left(\mathrm{T}_{3}\right)$ confirm the findings of Sarode and Gabhane (1994). They reported $27.25 \%$ fruit infestation of E.vittella with the treatment of NSE 5\%. The result on efficacy of neem oil $2 \%\left(\mathrm{~T}_{1}\right)$ agree with the findings of Malik and Lal (1989) and the result on efficacy of Bacillus thuringensis var. kurstaki $2 \mathrm{ml} / \mathrm{l}\left(\mathrm{T}_{4}\right)$ agree with the findings of Kharbade et al., (1999) and Singh et al., (1998). 
The treatment of Karanj oil 2\% $\left(\mathrm{T}_{2} ; 27.80 \%\right)$ found to be least effective but superior over control $\left(\mathrm{T}_{9} ; 38.70 \%\right)$. The result on Karanj oil $2 \%\left(\mathrm{~T}_{2}\right)$ confirm the results of Srinivasa rao et al., (2002) and Reddy and Srinivasa (2001) for brinjal shoot and fruit borer $L$. orbonalis.

Similar types of results were obtained 14 days after third spray in which the treatment of Spinosad 45 SC $0.01 \%\left(\mathrm{~T}_{8}\right)$ recorded the lowest (10.70\%) infestation. Next promising treatments were of Emamectin benzoate 5 SG $0.02 \%\left(\mathrm{~T}_{7} ; 21.20 \%\right)$, Indoxacarb $15.8 \mathrm{EC}$ $0.01 \%\left(\mathrm{~T}_{5} ; 22.77 \%\right)$, Acetamiprid 20 SP 0.2 $\mathrm{g} / \mathrm{lit}\left(\mathrm{T}_{6} ; 22.90 \%\right)$, Bacillus thuringensis 2 $\mathrm{ml} / \mathrm{l}\left(\mathrm{T}_{4} ; 24.10 \%\right)$ and these treatment were statistically at par with the treatment of Emamectin benzoate $5 \mathrm{SG} 0.02 \%\left(\mathrm{~T}_{7}\right)$

In botanicals promising treatments were of, Neem oil $2 \%\left(\mathrm{~T}_{1} ; 25.75 \%\right)$, NSE 5\% $\left(\mathrm{T}_{3}\right.$; $27.50 \%)$ and these treatments were statistically at par with each other.

The treatment regarding Karanj oil 2\% $\left(\mathrm{T}_{2}\right)$ showed higher infestation $30.07 \%$ but found to be superior over control $\left(\mathrm{T}_{9}\right)$, where $31.60 \%$ infestation was recorded.

\section{Cumulative per cent fruit infestation by shoot and fruit borer (on number basis)}

The cumulative per cent fruit infestation of all pickings presented in (Table 3 ) and illustrated in figure 2 revealed that, all the treatments were significantly superior over control $\left(\mathrm{T}_{9}\right)$. Among these different treatments, Spinosad $45 \mathrm{SC} 0.01 \%\left(\mathrm{~T}_{8}\right)$ was found to be the most promising one, which consistently maintained lowest fruit infestation throughout the period of experimentation with the average of $12.86 \%$ infestation. The next promising treatment was of Emamectin benzoate $5 \mathrm{SG}$ $0.02 \%\left(\mathrm{~T}_{7} ; 20.81 \%\right)$, Indoxacarb $15.8 \mathrm{EC}$ $0.01 \%\left(\mathrm{~T}_{5} ; 22.04 \%\right)$, Acetamiprid 20 SP 0.2 $\mathrm{g} / \mathrm{lit}\left(\mathrm{T}_{6} ; 22.46\right)$, Bacillus thuringensis $2 \mathrm{ml} / \mathrm{l}$
$\left(\mathrm{T}_{4}\right)$ in which $23.32 \%$ infestation was observed.

The remaining treatments in descending order of efficacy were, Neem oil $2 \%\left(\mathrm{~T}_{1} ; 24.32 \%\right)$ and NSE 5\% ( $\left.\mathrm{T}_{3} ; 25.39 \%\right)$ which were comparable with each other in reducing per cent fruit infestation. Our findings on NSE $5 \%\left(\mathrm{~T}_{3}\right)$ agree with the results of Singh et al., (1998). They recorded $27.84 \%$ fruit infestation 4 days after treatment NSE $(100 \%)$. The findings on efficacy of neem oil $2 \%\left(\mathrm{~T}_{1}\right)$ corroborate with the results of Singh et al., (1998). They recorded $27.98 \%$ fruit infestation of E.vittella with the treatment neem seed oil $(1 \mathrm{~kg} / \mathrm{ha})$. The results on efficacy of Bacillus thuringensis $2 \mathrm{ml} / 1\left(\mathrm{~T}_{4}\right)$ agree with studies of Singh et al., (1998). They reported $25.14 \%$ fruit infestation of E.vittella with the treatment of $B t$ Var. Kurstaki $1.5 \mathrm{~kg}$ a.i./ha.

The treatment Karanj oil 2\% $\left(\mathrm{T}_{2}\right)$ recorded $27.49 \%$ fruit infestation and this treatment was found to be least effective. The results on Karanj oil 2\% $\left(\mathrm{T}_{6}\right)$ could not be discussed for want of supporting literature.

\section{References}

Ambekar, J. S., A. S. Pawar, and M. V. Sakhare, 2001. Bio-efficacy of certain neem products against okra fruit borer, J. Maharastra Agri. Univ., 25 (1): 4243.

Anil and P. C. Sharma, 2010. Bioefficacy of insecticides against Leucinodes orbonalis on brinjal, Journal of environmental biology. 31, 399-402.

Anonymous, 2015. All-India Area, Production and Yield of okra. Directorate of Economics and Statistics, Department of Agriculture and Cooperation. http://www.mahaagri.gov.in. 
Anonymus, 2015. Indian horticulture database. National horticulture board, New Delhi at www.nhb.gov.in

Devi, L. L., M. Ghule, M. L. Chatterjee, and A. K. senapati, 2014. Biorational management of shoot and fruit borer of okra (Earias vittella Fabricius) and their effect on insect predators. Environment and Ecology 33 (3):1052-1054.

Kharbade, S. B., A.G. Chandele and C.S. Chauudhary, 1999. Comparative efficacy of Dipel 8L, Delfin WG and halt rock - 01 WP against Earias vitella. Pestology. 23(8): 51-52.

Malik, S.N. and I.B. Lal. 1989. Efficacy of neem oil cake and fertilizer mixture against okra fruit borer. Pestology. 13(11): 6-7.

Panzade D. S., 2006 Evaluation of some neem products and insecticides againsts $E$. vitella (Fab.) on okra M.Sc. Agri. Thesis submitted Dr. P.D.K.V. Akola.

Priya, B. S and H.P. Misra, 2007. Biopesticides for the management of okra fruit borer, Earias vittella (Fab) pest management in horticultural ecosystem, vol. 13 (2): pp 176-179.
Puranik, T.R., Hadapad, A.B., Salunkhe, G.N., D.S. Pokharakar, 2002. Management of shoot and fruit borer, Leucinodes orbonalis Guenee through Bacillus thuringiensis formulations on brinjal. J. Entomol. Res. 26(3): 229-232.

Reddy, E. and N. Srinivasa 2001. Efficacy of botanicals against brinjal shoot and fruit borer Leucinodes orbonalis Guen. Proceedings of National Symposium on Integrated Pest Management (IPM) in Horticultural crops: New Molecules Biopesticides and Environment, Bangalore 17-19, October, PP. 11-13

Sarode S. V. and A. T. Gabhane. 1994. performonce of neem seed kernal extract with reduced insecticidal dosages on the infestation of okra fruit borer, E. vitella (Fab.) J. Ent. Res. 18(4):327-330

Singh, G., S.C. Bhardwaj and G.S. Dhaliwal. 1998. Evaluation of some biopesticides for the management of fruit borer, Earias spp., on okra crop. Indian J. Ecol. 25(2): 187.189.

Srinivasa Rao, N. and R. Rajendran, 2002. Pest Management and Economic Zoology 10:131

\section{How to cite this article:}

Lakhamapure, A.R., Sh.N.V. Lavhe, P.S. Barde, R.O. Deotale and Panchabhai, P.R. 2018. Assessment of Shoot and Fruit Damage on Number Basis in Okra. Int.J.Curr.Microbiol.App.Sci. 7(12): 3513-3523. doi: https://doi.org/10.20546/ijcmas.2018.712.397 